\title{
Surface Patterson Function by Inversion of Low-Energy Electron Diffraction $I-V$ Spectra at Multiple Incident Angles
}

\author{
Huasheng $\mathrm{Wu}$ and $\mathrm{S}$. Y. Tong \\ Department of Physics, The University of Hong Kong, Hong Kong, China
}

(Received 27 October 2000; published 26 June 2001)

\begin{abstract}
An accurate Patterson function, free of artifacts, is obtained by transforming low-energy electron diffraction $I-V$ spectra at multiple incident angles. The demonstration is carried out using normal incidence measured spectra and calculated spectra at three angles of incidence. The errors between intensity spots in the Patterson function and known interatomic distances are less than $0.01 \AA$ in the horizontal direction and $0.09 \AA$ in the vertical direction. The reason for the high accuracy in the horizontal direction is given.
\end{abstract}

DOI: $10.1103 /$ PhysRevLett.87.036101

In x-ray crystallography, the Patterson function (PF) provides very important information relating to atomic positions within a three-dimensional unit cell of a crystal [1]. The Patterson function gives the vector positions of every atom relative to every other atom in the unit cell. In low-energy electron diffraction (LEED), the incident beam attenuates rapidly inside a solid due to strong inelastic damping. Thus, LEED intensity-energy $(I-V)$ spectra are very sensitive to the first three to five atomic layers of a solid. It has long been recognized that the strong multiple scattering of electrons by atoms in a crystal makes the recovery of a Patterson function from LEED $I-V$ spectra a very difficult task [2]. However, a recent demonstration by Chang et al. [3] using LEED $I-V$ spectra at normal incidence shows very promising results. In this Letter, we demonstrate that a sum of transforms of LEED $I-V$ spectra over multiple incident directions $\left(\vec{k}_{i}\right)$ and momenta transfers $\left(\vec{q}=\vec{k}_{f}-\vec{k}_{i}\right)$ can produce an artifact-free Patterson function of the surface region. By artifact-free, we mean each intensity spot in the Patterson function corresponds to a vector position of atomic pairs. We further show that the Patterson function is highly accurate in determining pairwise atomic distances in the direction parallel to the surface. This is due to cancellation of phase shift effects in the PF. Similar cancellations do not exist in electron holography.

LEED has the advantage that for a given $\vec{k}_{i}$, a set of $I-V$ spectra containing many beams (i.e., $\vec{k}_{f \|}=\vec{k}_{i \|}+$ $\left.\vec{g}_{\|}\right)$can be measured over a wide energy range, typically $50-400 \mathrm{eV}$, in a matter of a few minutes. Here, $\vec{g}_{\|}$is a two-dimensional reciprocal lattice vector (i.e., beam) of the sample. Thus, it is very convenient to vary $\vec{k}_{i}$ and measure different sets of $I-V$ spectra. The momentum transfer in terms of $\vec{g}_{\|}$is given by $\vec{q}=\left(\vec{g}_{\|}, q_{\perp} \hat{e}_{z}\right)$, where $\hat{e}_{z}$ is a unit vector normal to the surface of the sample. The Patterson function is obtained by a phase sum of the following integral:

$$
P(\vec{R})=\left|\sum_{\hat{k}_{i}} \sum_{\vec{g}_{\|}} \int I\left(\vec{k}_{i}, \vec{g}_{\|}+q_{\perp} \hat{e}_{z}\right) e^{i \vec{q} \cdot \vec{R}} d q_{\perp}\right|^{2} .
$$

PACS numbers: 68.35.Bs, 07.79.- v, 61.14.Hg, 68.55.-a

Because a typical LEED screen has a central opening of $\sim 9^{\circ}$ half-cone angle, the diffracted beams are collected within a half-cone angular range of $9^{\circ}-51^{\circ}$. This incomplete sampling of reciprocal lattice vectors at a single incident angle creates artifacts in the Patterson function. On the other hand, the reason why multiple scattering contributions are eliminated by the phase sum integral of Eq. (1) can be understood if one considers the scattered intensity in the far-field approximation $[4,5]$ :

$$
\begin{aligned}
I\left(\vec{k}_{i}, \vec{k}_{f}\right) \alpha & f_{1} e^{-\vec{q} \cdot \vec{r}_{1}}+f_{2} e^{-i \vec{q} \cdot \vec{r}_{2}} \\
& +f_{1} f_{3} e^{-i \vec{k}_{f} \cdot \vec{r}_{1}} \frac{e^{i k r_{13}}}{r_{13}} e^{i \vec{k}_{i} \cdot \vec{r}_{3}}+\left.\ldots\right|^{2} .
\end{aligned}
$$

In Eq. (2), surface atoms are located at $\vec{r}_{1}, \vec{r}_{2}$, etc. with respect to an arbitrary origin and $f_{i}$ is the scattering factor of atom $i$. The first two terms are single scattering events at $\vec{r}_{1}$ and $\vec{r}_{2}$, respectively, and the third term is a multiple scattering event wherein the incident electron scatters both at $\vec{r}_{1}$ and $\vec{r}_{3}$, with $r_{13}=\left|\vec{r}_{1}-\vec{r}_{3}\right|$. The cross term in Eq. (2) from single scattering events is

$$
I_{1,2}\left(\vec{k}_{i}, \vec{k}_{f}\right) \alpha f_{1} f_{2}^{*} e^{-i \vec{q} \cdot \vec{r}_{12}}
$$

where $\vec{r}_{12}=\vec{r}_{1}-\vec{r}_{2}$. At $\vec{R}=\vec{r}_{12}$, the phase $e^{-i \vec{q} \cdot \vec{r}_{12}}$ is exactly conjugate to that of the kernel $e^{i \vec{q} \cdot \vec{R}}$ of the integral in Eq. (1). Therefore, a maximum in the Patterson function is created at $\vec{R}=\vec{r}_{12}$. The phase of the cross term involving the multiple scattering event is different from that of the kernel:

$$
I_{1,3}\left(\vec{k}_{i} \vec{k}_{f}\right) \alpha f_{1} f_{1}^{*} f_{3}^{*} \frac{e^{-i k r_{13}}}{r_{13}} e^{i \vec{k}_{i} \cdot \vec{r}_{13}} .
$$

By varying $k$ and $\vec{k}_{i}$, the contribution from this cross term containing multiple scattering can be eliminated.

In the following, we demonstrate that an artifact-free Patterson function can be obtained by using a wide sampling of wave numbers $k$ and directions $\vec{k}_{i}$. The system we choose for demonstration is $\mathrm{Si}(111) \sqrt{3} \times \sqrt{3} R 30^{\circ}$-Ga. 
By conventional (trial-and-error) dynamical LEED analysis, the surface structure of this system has been determined [6,7]. A recent dynamical LEED study [7] has obtained an excellent van Hove-Tong $R$ factor [8] of 0.14 , using normal incidence $I-V$ spectra in the $50-400 \mathrm{eV}$ energy range. In Fig. 1, we show a schematic diagram of the structure of this system, with atoms in the surface region numbered from 1 to 29 . Figure 2 shows the Patterson function obtained by inverting measured normal incidence LEED spectra of 150 beams (30 independent beams due to symmetry of the system). The figure shows that each vector distance between atomic pairs corresponds to a dark spot in the Patterson function. The atomic pairs, corresponding to the atom numbers shown in Fig. 1, are indicated next to the spots. The upper panel shows the Patterson function in the $(2 \overline{1} \overline{1})$ cross-sectional plane, which cuts through, among other atoms, atoms 1, 5, 6, and 9 of Fig. 1. The lower panel shows the (101) cross-sectional plane, which cuts through atoms 1, 4, 6, and 9, etc. of Fig. 1. Unlike previous inversions [9-12], we have used the intensities $I\left(\vec{k}_{i}, \vec{k}_{f}\right)$ in the transform of Eq. (1), and not the normalized functions $\chi\left(\vec{k}_{i}, \vec{k}_{f}\right)=I\left(\vec{k}_{i}, \vec{k}_{f}\right) / I_{0}\left(\vec{k}_{i}, \vec{k}_{f}\right)-1$. The only normalization included before the transform is that the LEED intensities are divided by a constant number. This constant number is simply the average of intensities over all energies and beams. We find that the transform of intensities, rather than normalized functions, produces brighter spots for atomic pairs that are parallel to the surface. The wave number inside the solid is used in Eq. (1), i.e., $k=\sqrt{2 m / \hbar^{2}\left(E+V_{0}\right)}$, where $V_{0}$ is an average inner potential taken to be $5 \mathrm{eV}$ in all materials. To suppress spurious noise near the origin, the transform inside a hemisphere of radius $1.5 \AA$ from the origin is set to zero. The intensities are multiplied by a factor of $E^{2}$ to compensate for the decrease due to the scattering factor and the Debye-Waller factor. In electron holography, the $\chi$ function is used because there is a reference atom with

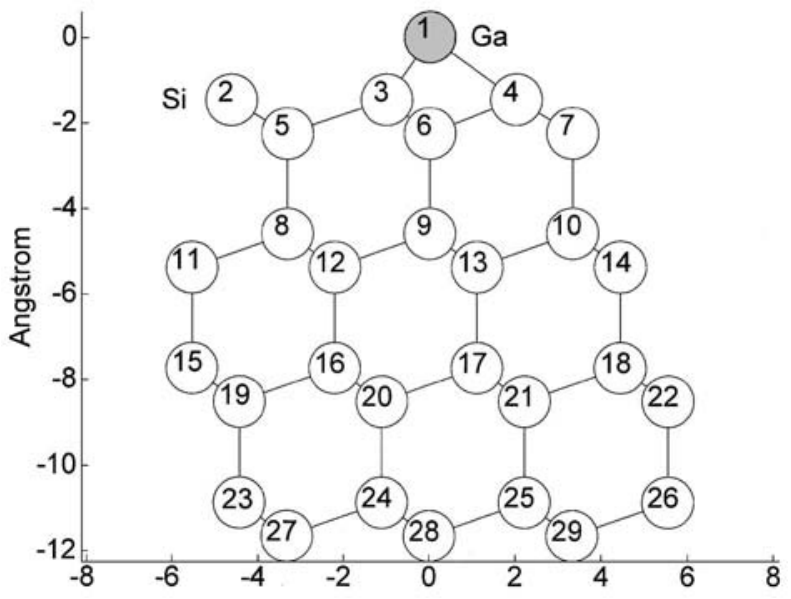

FIG. 1. Schematic view of $\mathrm{Si}(111) \sqrt{3} \times \sqrt{3} R 30^{\circ}$-Ga with $\mathrm{Ga}$ at the $\mathrm{T}_{4}$ site and $1.46 \AA$ above the topmost $\mathrm{Si}$ atoms. a direct wave. In LEED, there is no reference atom. The first order term is the interference of single scatterings from two atoms. Thus, the x-ray or LEED PF uses directly the intensities and not the $\chi$ functions.

In both panels of Fig. 2, there are many dark spots which do not correspond to any interatomic vector distance. These other spots, or "artifacts," are caused by either an incomplete sampling of the two-dimensional reciprocal lattice space or the failure of the transform to eliminate all strong multiple scattering contributions. To demonstrate the effect of multiple scattering on the Patterson function more directly, we show in Fig. 3 the Patterson function obtained by inverting calculated LEED $I-V$ spectra using multiple scattering theory [7], under identical conditions as the experiment. Because of the close agreement between measured and calculated $I-V$ curves (low van Hove-Tong $R$ factor), it is not surprising that there are strong resemblances between the Patterson functions recovered from measurement and multiple scattering calculations. Even the artifacts appear at approximately similar locations. However, the main conclusion to be drawn from examining Figs. 2 and 3 is that strong multiple scattering contributions are not eliminated in the transforms of just normal incidence LEED $I-V$ spectra, either from measurement (Fig. 2) or calculation (Fig. 3).

It is possible, however, to obtain a Patterson function that is completely free of artifacts. This is accomplished
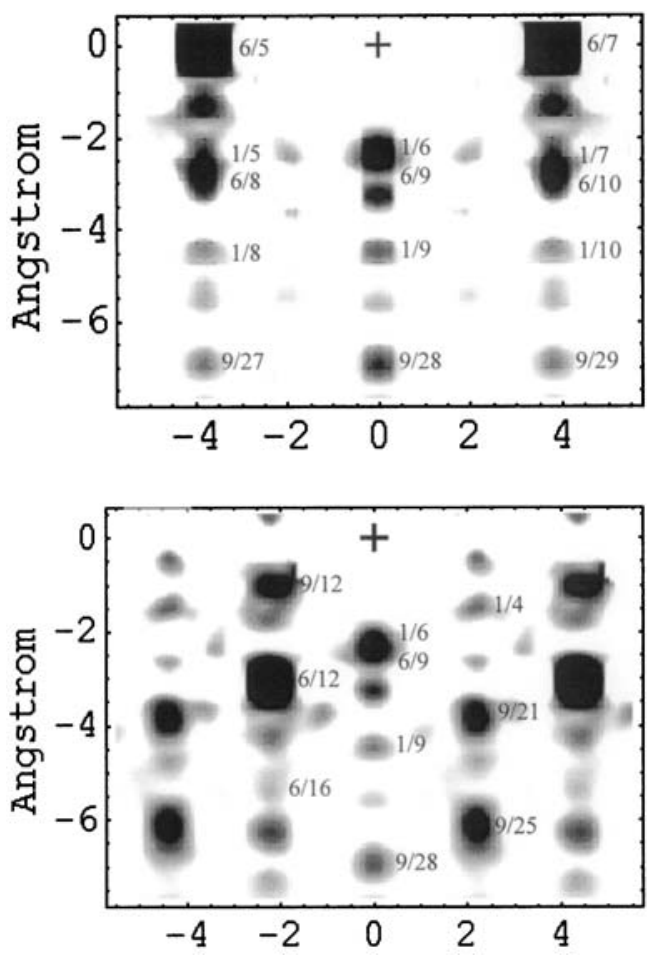

FIG. 2. Patterson function from normal incidence experimental $I-V$ spectra: the upper panel shows the $(2 \overline{1} \overline{1})$ plane; the lower panel shows the (101) plane. In each $x y$ plane, which is parallel to the surface, the lowest $10 \%$ contours are not shown. The Patterson function contains many artifacts. 

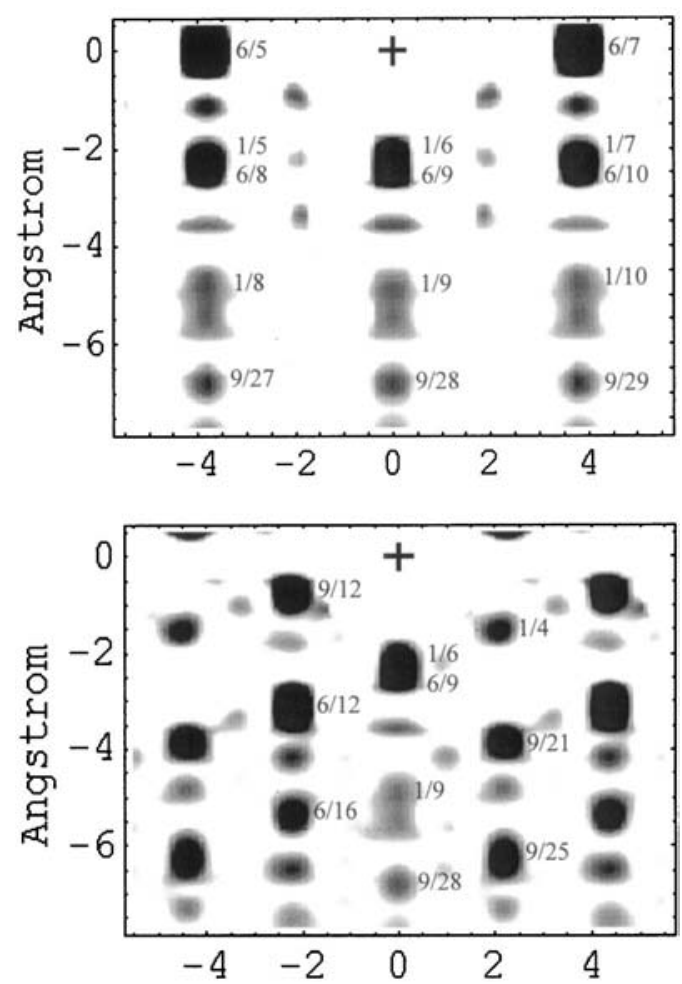

FIG. 3. Patterson function from normal incidence calculated $I-V$ spectra using multiple scattering theory. Other conditions are the same as in Fig. 2. The Patterson function contains many artifacts.

by varying $\vec{k}_{i}$. It is easy to see from Eq. (4) that as $\vec{k}_{i}$ is varied, the exponential factor in the cross term containing the multiple scattering event will change. The effect is a more complete phase cancellation of multiple scattering terms. Furthermore, a different $\vec{k}_{i}$ allows additional reciprocal lattice vectors to be added to the sampling range. We show in Fig. 4 the sampling of $\vec{k}_{f}$ using three incidences, i.e., $\theta=0^{\circ} ; \theta=20^{\circ}, \phi$ along $[11 \overline{2}]$; and $\theta=20^{\circ}, \phi$

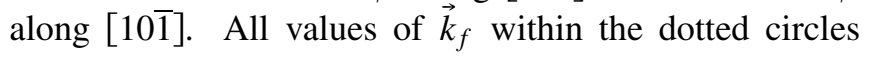
are sampled. Since the system has a $C_{3 v}$ symmetry, a rotation of $120^{\circ}$ is exercised on each set of off-normal incidence. Finally, Fig. 5 shows the Patterson function

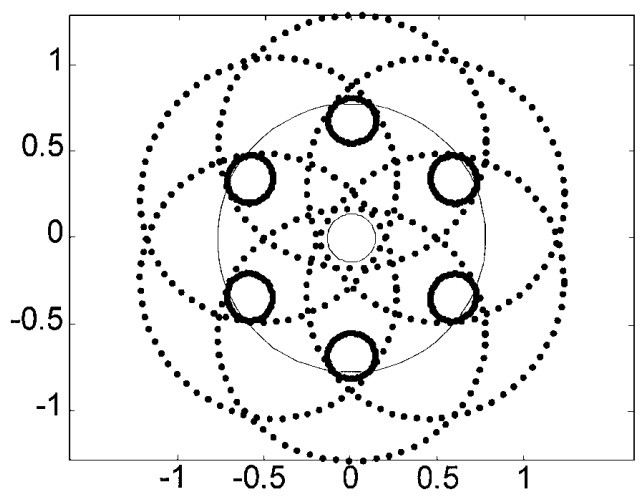

FIG. 4. Reciprocal lattice space $\vec{q}_{\|} / k$ using normal and $\theta=$ $20^{\circ}$ incident angles and $\phi$ along mirror planes. The small circles represent the central opening of the LEED detector. obtained from inverting $I-V$ spectra calculated by multiple scattering theory using the incident directions shown in Fig. 4. The Patterson function so obtained is completely artifact-free; every spot corresponds to an interatomic vector distance. More significant is the fact that the Patterson function is very accurate. Table I lists the interatomic distances obtained from the atom positions used in the multiple scattering calculation, i.e., $x_{0}$ and $z_{0}$, and the positions of the spots of the Patterson function of Fig. 5, i.e., $x$ and $z$. The average error in the vertical distances is only $\langle|d z|\rangle=\left\langle\left|z-z_{0}\right|\right\rangle=0.03 \AA$, with the largest error being $0.09 \AA$ A The average error in the horizontal distance is even smaller, most spots in the Patterson function occur at exactly the $x_{0}$ position, with the largest error being only $0.01 \AA$. Although the Patterson function in Fig. 5 is obtained from $I-V$ curves calculated by multiple scattering theory, it nevertheless indicates an important application of the method that is complementary to the trial-and-error approach. In the trial-and-error approach with near normal angle $I-V$ spectra, the sensitivity to horizontal distances is low and their error bars are large $\sim 0.15-0.20 \AA$. This in turn leads to large uncertainties in the surface bond lengths. Since the Patterson function shows a high accuracy in horizontal distances, it provides important new information of the surface structure. As an example, the atomic pair $(1,4)$ is between the $\mathrm{Ga}$ atom and $\mathrm{Si}$ atom 4 in Fig. 1. In the calculation, the $\mathrm{Si}$ atom 4 is shifted towards the $\mathrm{Ga}$ atom horizontally by $0.20 \AA$, resulting in $x_{0}=2.02 \AA$, instead of the bulk value $2.22 \AA$. The spot (1/4) in the Patterson function of
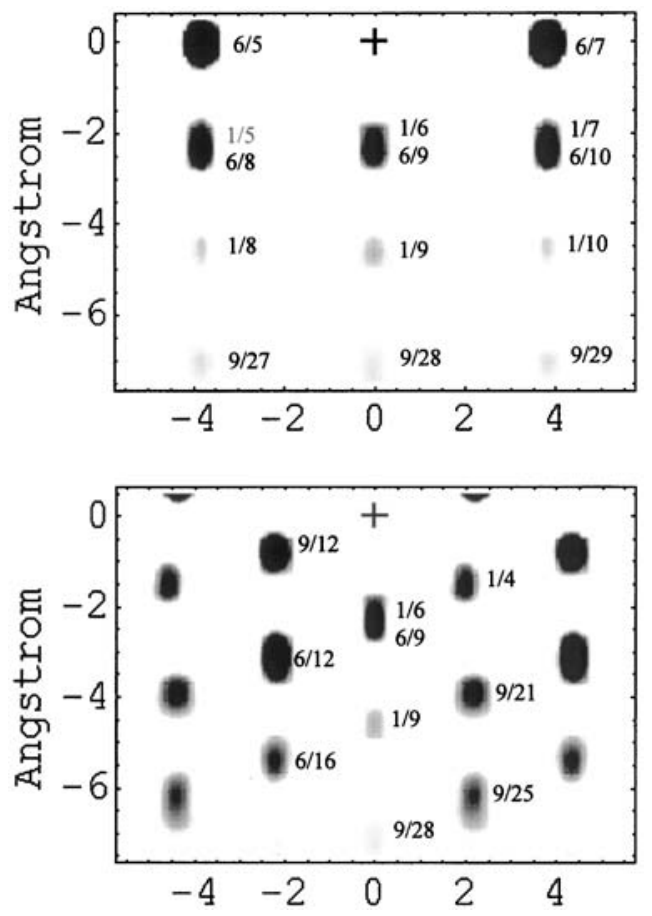

FIG. 5. Patterson function from $I-V$ spectra of multiple incident angles as those shown in Fig. 4, using multiple scattering theory. Other conditions same as in Fig. 2. The Patterson function is artifact-free. 
TABLE I. Interatomic distances in $\AA$ unit, origin at the $\mathrm{Ga}$ atom, in the horizontal $\left(x_{0}\right)$ and vertical $\left(z_{0}\right)$ directions using atomic positions of multiple scattering calculation; the corresponding quantities $(x, z)$ using spot positions of the Patterson function in Fig. 5 , and their differences $(d x, d z)$. Atomic pairs $1 / 6$ and $6 / 9$ are too close to be resolved and the average value is used for $x_{0}$ and $z_{0}$. Similarly for atomic pairs $1 / 7$ and $6 / 10$.

\begin{tabular}{|c|c|c|c|c|c|c|}
\hline Atomic pairs & $x_{0}$ & $z_{0}$ & $x$ & $z$ & $d x$ & $d z$ \\
\hline $1 / 6,6 / 9$ & 0.00 & -2.30 & 0.00 & -2.32 & 0.00 & -0.02 \\
\hline $1 / 9$ & 0.00 & -4.59 & 0.00 & -4.62 & 0.00 & -0.03 \\
\hline $9 / 28$ & 0.00 & -7.04 & 0.00 & -7.00 & 0.00 & 0.04 \\
\hline $6 / 7$ & 3.84 & 0.00 & 3.84 & 0.00 & 0.00 & 0.00 \\
\hline $1 / 7,6 / 10$ & 3.84 & -2.30 & 3.84 & -2.30 & 0.00 & 0.00 \\
\hline $1 / 10$ & 3.84 & -4.59 & 3.84 & -4.50 & 0.00 & 0.09 \\
\hline $9 / 29$ & 3.84 & -7.04 & 3.85 & -7.00 & 0.01 & 0.04 \\
\hline $9 / 12$ & -2.22 & -0.78 & -2.23 & -0.76 & -0.01 & 0.02 \\
\hline $6 / 12$ & -2.22 & -3.13 & -2.23 & -3.10 & -0.01 & 0.03 \\
\hline $6 / 16$ & -2.22 & -5.48 & -2.22 & -5.41 & 0.00 & 0.07 \\
\hline $1 / 4$ & 2.02 & -1.46 & 2.02 & -1.50 & 0.00 & -0.04 \\
\hline $9 / 21$ & 2.22 & -3.91 & 2.22 & -3.90 & 0.00 & 0.01 \\
\hline $9 / 25$ & 2.22 & -6.26 & 2.22 & -6.20 & 0.00 & 0.06 \\
\hline
\end{tabular}

Fig. 5 is correspondingly shifted horizontally towards the Ga atom, giving $x=2.02 \AA$.

Patterson function differs from electron holography in that $\mathrm{PF}$ is the inversion of the cross term between two first order scattering events [given in Eq. (3), i.e., $f_{1} f_{2}^{*} e^{-i \vec{q} \cdot \vec{r}_{12}}$ ]. Electron holography, on the other hand, is the inversion of the cross term between a reference wave and a direct wave scattered by a nearby atom. For pairwise distances of the same kind of atoms (e.g., Si-Si distances), the contribution from the scattering factors $f_{1} f_{2}^{*}$ in PF exactly cancels. No such cancellation occurs in electron holography because its leading term contains a single factor of $f_{1}$ $[4,5]$ and not a product of $f_{1}$ with the complex conjugate $f_{2}^{*}$. Furthermore, the LEED geometry always collects data in the backscattering direction, even at off-normal incidences. In the backscattering geometry, the difference between backscattering factors of different elements is small. To explain the high accuracy of the PF in determining horizontal pairwise distances, we note that the LEED data are taken under symmetric $\vec{q}=\vec{k}_{f}-\vec{k}_{i}$, i.e., using mirror and rotation symmetries as shown in Fig. 4. The shifts of atomic distances $[4,5]$ in directions parallel to the surface caused by $f_{1} f_{2}^{*}$ of different elements exactly cancel. This is because for each term containing $f_{1}\left(\vec{q}_{\|}\right) f_{2}^{*}\left(\vec{q}_{\|}\right)$, a term containing $f_{1}\left(-\vec{q}_{\|}\right) f_{2}^{*}\left(-\vec{q}_{\|}\right)$is also included. The same is not true in electron holography, where the scattering angle is between the bond direction $\vec{r}_{j}$ and $\vec{k}_{f}$ [4,5]. While $\vec{k}_{f}$ can be symmetrized, the bond direction $\vec{r}_{j}$ is fixed. As Table I shows, the errors $d x$ are $0.01 \AA$ or less. Indeed, $d x$ in Table I is caused by the limited $k$ space sampling and not by phase shifts of the scattering factor.

The complementary nature of the trial-and-error approach and the Patterson function approach cannot be overemphasized. In the former, a "best" structure is determined from an automated search over an extensive set of trial structures. However, even if the best structure has a low $R$ factor, there is still no guarantee that another structure might not produce an even lower $R$ factor. The Patterson function, on the other hand, is obtained directly from LEED $I-V$ spectra without structural modeling. Once an accurate Patterson function is obtained, the interatomic distances obtained from the best structure of the trial-and-error approach must fit the spots of the Patterson function; otherwise the best structure cannot be correct.

We acknowledge helpful discussions with Geng Xu, Simon Ma, and Russell Fung. The support of RGC Grants No. HKU7117/98P, No. HKU7120/00P, No. HKU7396/ 00P, DOE Grant No. DE-FG02-84ER45076, and NSF Grant No. DMR-9972958 are acknowledged.

[1] A. L. Patterson, Phys. Rev. 46, 372 (1934).

[2] David L. Adams and Uzi Landman, Phys. Rev. B 15, 3775 (1977).

[3] C. Y. Chang, Z. C. Lin, Y.C. Chou, and C. M. Wei, Phys. Rev. Lett. 83, 2580 (1999).

[4] S. Y. Tong, Adv. Phys. 48, 135 (1999).

[5] S. Y. Tong, H. Li, and H. Huang, Surf. Rev. Lett. 1, 303 (1994).

[6] A. Kawazu and H. Sakama, Phys. Rev. B 37, 2704 (1988).

[7] Wenhua Chen, Huasheng Wu, Wing Kin Ho, B. C. Deng, Geng Xu, and S. Y. Tong, Surf. Rev. Lett. 7, 267-270 (2000), and references therein.

[8] M. A. Van Hove, S. Y. Tong, and M. H. Elconin, Surf. Sci. 64, 85 (1977).

[9] D. K. Saldin, Surf. Rev. Lett. 4, 441 (1997).

[10] S. Y. Tong, H. Huang, and C. M. Wei, Phys. Rev. B 46, 2452 (1992).

[11] C. M. Wei and S. Y. Tong, Surf. Sci. 274, L577 (1992).

[12] S. Y. Tong, Surf. Sci. 433-435, 32 (1999). 